\title{
Women's Responses and Understanding of Polygenic Breast Cancer Risk Information
}

Yanes T., ${ }^{1,2}$ Kaur R., ${ }^{1}$ Meiser B., ${ }^{1}$ Scheepers-Joynt M., ${ }^{3}$ McInerny S., ${ }^{3}$ Barlow-Stewart K., ${ }^{4}$ Antill Y., ${ }^{5}$ Salmon L., ${ }^{6}$ Smyth C., ${ }^{7}$ James P.A. ${ }^{3,8}$ Young M.A. ${ }^{3,9}$

${ }^{1}$ Prince of Wales Clinical School, Faculty of Medicine, University of New South Wales, Sydney NSW 2052, Australia

${ }^{2}$ School of Psychiatry, Faculty of Medicine, University of New South Wales, Sydney NSW 2052, Australia

${ }^{3}$ Parkville Familial Cancer Centre, Peter MacCallum Cancer Centre and the Royal Melbourne Hospital, Melbourne VIC 3000, Australia

${ }^{4}$ Northern Clinical School, Faculty of Medicine and Health, University of Sydney, Sydney NSW 2065, Australia

${ }^{5}$ Family Cancer Clinic, Cabrini Health, Melbourne VIC 3144, Australia

${ }^{6}$ Clinical Genetics Service, Austin Hospital, Melbourne VIC 3084, Australia

${ }^{7}$ Family Cancer Clinic, Monash Medical Centre, Melbourne VIC 3168, Australia

${ }^{8}$ Sir Peter MacCallum Department of Oncology, University of Melbourne, Melbourne VIC 3052, Australia

${ }^{9}$ Kinghorn Centre for Clinical Genomics, Garvan Institute of Medical Research, Sydney NSW 2010, Australia

Corresponding Author: Tatiane Yanes, Psychosocial Research Group, Lowy Cancer Research Centre C25, The University of New South Wales, UNSW Sydney, NSW 2052, Australia. Email: t.yanes@student.unsw.edu.au Fax: +61 (2) 9385 0033. ORCID ID: 00000002-3905-3025

Key words: breast cancer, qualitative research, polygenic risk scores, psychosocial research 


\section{Abstract:}

It is estimated that polygenic factors can explain up to $18 \%$ of familial breast cancer. Clinical implementation of polygenic testing has begun, with several commercial laboratories now testing. Despite commercial implementation, there is little research investigating how women respond and understand polygenic risk information. This study aimed to explore women's experience receiving their personalized polygenic risk score (PRS) and compare responses of women at different levels of polygenic risk. Eligible participants were affected and unaffected women from families clinically assessed to be at high risk for breast cancer who had received their personalized PRS as part of the Variants in Practice Psychosocial Study (ViPPs). In-depth semi-structured interviews were conducted with 21 women (mean age 53.4 years) up to four weeks after receiving their PRS. Interviews were transcribed verbatim and analyzed using thematic analysis. Eleven women received a PRS that was in the top quartile of PRS distribution and 10 in the lowest quartile. Women's lived experience with breast cancer informed how they responded to their PRS, constructed and made sense of breast cancer risk following receipt of their PRS, and integrated this new information into their breast cancer risk management. Regardless of polygenic risk level, all participants demonstrated broad knowledge of concepts related to polygenic information and were able to accurately describe the implications of their PRS. Receiving PRS did not appear to negatively impact women's reported distress levels. Our findings suggest polygenic breast cancer information is well received and understood by women at high-risk for breast cancer. 


\section{Introduction:}

It is estimated that a pathogenic variant in a high or moderate breast cancer risk gene is identified in less than $25 \%$ of families at high risk for the disease [1], meaning that for the majority of women whose family history suggest high risk, the etiology remains unexplained. Genome wide associations studies have identified over 180 single nucleotide polymorphisms (SNPs) associated with breast cancer risk [2, 3]. Individually, each SNP has a minimal impact on breast cancer risk. However, their combined effect (polygenic risk) is associated with at least a two-fold increased risk for breast cancer and increased risk for contralateral breast cancer [4-6]. It is estimated that polygenic factors explain an additional $18 \%$ of the genetic contribution to familial breast cancer risk [2]. Therefore, testing for breast cancer SNPs and computing a risk score has the potential to provide personalized breast cancer risk information to a significant proportion of women from high-risk breast cancer families. Implementation of polygenic testing has begun, with several commercial genetic testing laboratories now offering testing $[7,8]$.

Despite commercial implementation, there are limited studies assessing women's response to polygenic breast cancer risk information. Previous studies have focused on women with a personal history of breast cancer whose results showed they were in the top quartile of polygenic risk distribution $[9,10]$. These studies reported women felt positive about receiving their personalized breast cancer polygenic risk score (PRS), experienced little adverse psychological outcomes, and were able to integrate their PRS into their existing breast cancer risk perceptions. Only a small number of women reported feeling anxious about their PRS, primarily as it reawakened memories from their breast cancer diagnosis [9]. 
Polygenic risk information has also been assessed in other familial cancer setting, namely among unaffected men with a family history of prostate cancer and no known hereditary cancer diagnosis $[11,12]$. The men who received PRS for prostate cancer risk reported little impact on their emotional state, were able to accurately recall their personalized cancer risk, and felt reassured by the information $[11,12]$. However, for some participants, fatalistic beliefs about prostate cancer remained even after a low polygenic risk result. Instead risk perceptions were largely shaped by their family experiences of the disease. Receiving polygenic risk information had little impact on participants' cancer screening intentions [12]. As polygenic testing is implemented more broadly into clinical practice, it is important to understand how women respond to this information. This study invited women with (affected) and without (unaffected) a personal history of breast cancer to receive their individual breast cancer PRS. Previous genetic testing results in their family had been uninformative. This study aimed to explore responses of women who received their PRS and compare the responses of women at different levels of polygenic risk.

\section{Methods:}

\section{Genetic Testing and Participants:}

Participants were recruited from an established cohort, the Variants in Practice (ViP) study [13]. The ViP cohort is comprised of women affected by breast cancer and their unaffected relatives. Prior to enrollment in ViP all families had undergone a clinical assessment and genetic testing at a familial cancer clinic (FCC) in the Australian states of Victoria and Tasmania. Additional genotyping of 62 breast cancer associated SNPs was conducted 
through the ViP study, from which individual PRS and relative risk (RR) for breast cancer were generated.

This qualitative study was conducted as part of the Variants in Practice Psychosocial study (ViPPs) [14]. The ViPPs is a mixed-methods study that aims to assess the important psychosocial and behavioral outcomes associated with receiving breast cancer PRS. Eligible women were sent a study invitation package that included a participant information and consent sheet, a response form, and a two-page educational pamphlet on genomic testing and breast cancer risk [15]. Eligibility included: women currently enrolled in ViP, aged 18 years or older, from a family with an uninformative genetic test result for high and moderate breast cancer risk genes and who had a PRS that was in the highest (high PRS) or lowest (low PRS) quartile of polygenic risk distribution. A purposive sampling strategy was employed for this qualitative study [16], with women at different levels of breast cancer polygenic risk invited to participate (high and low PRS). No exclusions were made based on personal history of breast cancer. This study was approved by the Peter MacCallum Cancer Centre (HREC/16/PMCC/2) and the Tasmanian Research Ethics Committee (H0016395).

\section{Genetic Counselling:}

The genetic counselling sessions to return the personalized breast cancer PRS were conducted as part of ViPPs by genetic health professional at participating FCCs in the Australian states of Victoria or Tasmania. The appointments included disclosure of the participant's personalized breast cancer PRS, a discussion about the implications of the PRS in line with participants' personal and/or family history of breast cancer and breast cancer risk management strategies. A graphical representation of each individual's PRS including 
their relative risk of breast cancer compared to the Australian general population and the impact of lifestyle factors (alcohol consumption and body mass index greater than 30) were used when explaining PRS results. Reporting of polygenic results has been described elsewhere [10].

\section{Data Collection and analysis:}

In-depth interviews were conducted with participants up to four weeks after they received their PRS. An interview guide based on the literature and the research aims was used and explored the participants' experiences with breast cancer, recall and understanding of PRS, communication of results, feelings about breast cancer risk, and breast cancer risk management. A genetic counsellor (TY) who was not involved in the return of PRS results conducted the research interviews by telephone between June 2017 and October 2018. With consent, all interviews were audio-recorded, transcribed verbatim and pseudonyms assigned. Interviews continued until data saturation was reached for the entire sample [17].

A thematic analysis was conducted using the approach described by Braun and Clarke [17]. This iterative process involves systematically identifying, comparing and coding themes within and across interviews and accounts for deviant cases. Emerging patterns and relationships between the codes led to the development of second-order categories. Analytical rigor was achieved by other members of the research team (MAY and RK) reading the interviews to confirm the coding and themes. Interviews were coded using NVivo11 [18].

\section{Results:}


Out of the 23 women invited to take part in this qualitative study, 2 declined with 21 participating in a telephone interview. Interviews ranged from 20 minutes to 90 minutes in length (mean 43 minutes). Participants were aged between 38 to 77 years (mean age 56 years SD 13.3) and most women were born in Australia (85\%), spoke English at home (100\%) and had completed a bachelor's degree or above (62\%) (Table 1). Eleven women had a personal history of breast cancer (mean age diagnosis 48 years, SD 8.07) and 10 were unaffected. Out of the unaffected women five received a high PRS and five a low PRS. Of the affected women, six had a high PRS and five had a low PRS. All affected participants had attended an FCC in the past and had BRCA1/2 genetic testing, which was uninformative. Among unaffected participants, all had at least one first-degree relative diagnosed with breast cancer and most $(90 \%)$ had attended an FCC to discuss their family history of breast cancer. Relative risk for breast cancer ranged from 0.30 to 3.37 for affected women and 0.50 to 2.10 for unaffected.

Four key themes were developed: The decision to receive PRS, Response to PRS, Making sense of breast cancer risk, and Impact on breast cancer risk management. Informing these themes was participants' lived experience of breast cancer. Their lived experience influenced how women responded to their personalized PRS, constructed and made sense of breast cancer risk following receipt of their personalized PRS, and integrated this new information into their breast cancer risk management.

\section{The Decision to Receive PRS:}


For most participants the decision to receive their PRS was straightforward. In fact, most participants spoke positively about the invitation to receive their PRS, with many describing it as an opportunity.

"It wasn't a really big decision at all, but it just felt like it was the right thing to do." (Donna, 40 years, Affected/Low PRS)

"I wouldn't have thought of not doing it. When, you know, when you're offered that opportunity, most definitely. ” (Rebecca, 52 years, Unaffected/High PRS)

Only a small number of participants reported having concerns prior to receiving their PRS, including an adverse emotional response to a high-risk result, implications for family members, and insurance. However, these concerns were reportedly often short-lived, with many reflecting their concerns were outweighed by their desire to access information.

"I did hesitate thinking do I want to know if it's a year away that I might get it again?

And I thought no...I want to know either way. " (Sally, 77 year, Affected/Low PRS)

'Every now and then I'd think oh what if she says that, you know, we've got a really high risk? But then I thought well if that's the case it's better to know" (Lara, 59 years, Unaffected/High PRS)

\section{Factors influencing decision to receive PRS:}

Participants provided a range of reasons for why they decided to receive their PRS, which included curiosity and helping research. However, for nearly all participants this decision was based on their beliefs that "knowledge is power". This attitude was reflective of women's coping style, with many describing they would rather have information than not know. 
"...for me information is never bad regardless of what that information contains, whether it's positive or negative information the - the acquiring of information to me is always a very positive thing..." (Amanda, 47 years, Affected/High PRS)

"Yeah, just that I'm one of those people who I'd rather know than not know" (Carly, 45 years, Unaffected/Low PRS)

Among unaffected women, most described being motivated to receive their PRS due their family history of breast cancer.

"My history is so huge, it's a no-brainer. Find out, prevent it" (Lara, 59 years, Unaffected/High PRS)

In addition, unaffected participants often reflected on their relatives' uninformative $B R C A 1 / 2$ result. These participants expressed uncertainty about their risk level, and in some cases struggled to make decisions on how best to manage their breast cancer risk. Many unaffected participants were therefore driven to receive their PRS out of a desire to have greater certainty regarding their risk level, even if that meant being told they were at higher risk for breast cancer.

“it's been so kind of you should have MRI's, oh no you shouldn't, you should have this screening...oh you have got this lump and it's probably nothing. You know there's heaps of uncertainty around it [breast cancer risk]... as I said to my partner on the way in there, I hope they just tell me high-risk and I can just have a solid answer, so I kind of wanted certainty from it..." (Brittany, 36 years, Unaffected/Low PRS)

Whilst affected participants also hoped to have greater certainty regarding their risk level, they appeared primarily motivated to obtain information to explain their previous breast 
cancer diagnosis. For these women, their breast cancer diagnosis had been a traumatic event, one they could not explain given their uninformative genetic testing result.

“...I really was interested to know if they found out anymore and whether or not I'll

fit it in to that category of new knowledge that would I guess explain what happened

for me." (Sam, 59 years, Affected/High PRS)

“The old saying 'Why me?” (Sharon, 53 years, Affected/Low PRS)

Finally, regardless of personal history, participants reported receiving their PRS due to a sense of responsibility for their family, particularly their female relatives.

“...but also I've got a daughter and two granddaughters now so as much information as we can get." (Kelly, 66 years, Affected/Low PRS)

"I've got a young - a daughter, she's just turned 26, so whatever I'm doing now is really to her in the future." (Emily, 57 years, Unaffected/High PRS)

\section{Responses to PRS:}

Initial responses to PRS varied and included relief, surprise and even some reassurance. However, it was evident that participants' initial responses were significantly impacted by their lived experience of breast cancer and the PRS they received.

High PRS:

All unaffected participants who received a high PRS described not being surprised by their result. These participants reflected on their family history of breast cancer and felt their PRS was merely a confirmation of their existing risk perceptions. Similarly, all affected 
participants with a high PRS described not being surprised by this result as this was aligned with their prior experiences with the disease.

“...because I had a triple negative cancer that wasn't BRCA1 or BRCA2, I knew that there was a - I knew that there was something else..." (Amanda, 47 years, Affected/High PRS)

"I expected that [high PRS]...because of my family history on both sides of the family, having different cancers in the past.” (Elisa, 52 years, Unaffected/High PRS)

Affected and unaffected participants who had previously attended an FCC also recalled discussions with their health care providers about the possibility of other genetic risk factors being associated with their personal and/or family history of breast cancer. These participants felt they had been informed about ongoing research and the possibility of uncovering new genetic information that could explain their personal and/or family history of breast cancer.

“...I didn't have those two genes [BRCA1/2] but the genetic counsellor I talked to way back then...he said it would be very surprising if there wasn't gene involvement somewhere. So I've always sort of had this sort of idea that that's probably quite likely” (Judy, 43 years, Affected/High PRS)

Participants also felt positive about their PRS and valued the additional information provided to them. For example, unaffected participations were relieved to have access to information to help them make sense of their breast cancer risk and felt positive about having greater certainty about their risk level. 
“Before it was you may have a risk, you know, they've taken that may away...it's now given me that tool to go, okay, I didn't know, but now I do know, it's just another tool in the toolbox". (Emily, 57 years, Unaffected/High PRS)

Affected participants also valued having an etiological explanation for their cancer, with some describing their result had absolved them from past blame for their diagnosis.

“...we had a bad car accident...and the seatbelt came very sharply across my left breast...So, there was always did it [breast cancer] happen because of that? Is it just a fluke? But when I got my results back, I knew it wasn't a fluke." (Jessica, 66 years, Affected/High PRS)

Despite all participants with a high PRS not being surprised by their result, some affected women expressed feeling initially confronted by their PRS as it reawaked the trauma from their breast cancer diagnosis and was a confirmation of their increased risk for contralateral breast cancer.

"I suppose this is a bit more confronting because one, it's confirmed what I'd thought [increased breast cancer risk] and secondly, I have to think back to that time, even just what was my diagnosis, what did I have done, all things that I try not to think about. It brings things back.” (Isabel, 49 years, Affected/High PRS)

In contrast, no unaffected participant with a high PRS reported feeling confronted by their breast cancer risk. This response was often based on their experiences with breast cancer, which included limited experience with an affected relative, or relatives for whom their cancer was treatable. 
"I'm fine. I can be vigilant and it's not alarming particularly...I mean I know you hear about obviously people die of breast cancer but my mother, she seems to know a lot of people who have had or got it and a lot of people do have a good prognosis and do have treatment and then are okay. I mean she's a perfect example too so I figure that obviously if there's any early detection then it's nipped in the bud these days pretty quickly hopefully.” (Rebecca, 52 years, Unaffected/High PRS)

\section{Low PRS:}

Responses from women who received a low PRS were more straightforward. Unaffected participants described their result as "good news" and often felt reassured by their PRS result, with many reflecting that no pathogenic variant had been identified after extensive genetic testing in their family.

"Getting these results was actually good I guess for me because it just clarified again that even with the new medical information available, still genetically it doesn't look like I'm any more likely to get breast cancer than anyone else in the population." (Carly, 45 years, Unaffected/Low PRS)

Despite all unaffected participants feeling positive about their result, some women with a low PRS struggled to reconcile the differences between their PRS and their family history of breast cancer. These participants felt they needed additional information in order to put their result into context, including knowing their affected relative's PRS.

"It was a happy result for me, an unexpectedly happy result, but I can't sort of put it into any context unless I know what my mum's bell curve looked like” (Ashely, 42 years, Unaffected/Low PRS) 
Similarly, affected participants described their low PRS as a positive result.

"I've got a reason to be quite relieved about the results" (Barbara, 75 years/Low PRS)

“When I got the result, going home, yeah, I certainly felt much better." (Sharon, 53 years, Affected/Low PRS)

However, they acknowledged their low PRS did not explain their personal history of breast cancer. These participants said they would continue searching for answers but were also accepting of the fact that they may never have an explanation for their breast cancer.

“it still remains a question of why did I get breast cancer? So it doesn't really make too much of a difference. I think once you go through something like this and there's no formal, no family history then it's just always going to be a question. I'm hoping that they find an answer to why it is but I'm also realistic that they may not. So we'll just do what I can do and be part of whatever I can be part of until then." (Donna, 40 years, Affected/Low PRS)

Despite differences in participants' initial reactions, receiving a PRS did not appear to have a significant impact on their emotional state. In fact, all participants, regardless of their personal history or whether they received a high or low PRS, reported having come to accept this new information in the weeks since receiving their result with many saying "I haven't been stressing about it" (Amanda, 47 years, Affected/High PRS); "I haven't been worried about it" (Emma, 50 years, Unaffected/High PRS), "I don't dwell on it" (Ashely, 42 years, Unaffected/Low PRS) and "I'm not worried" (Barbara, 75 years/Low PRS).

\section{Making Sense of Breast Cancer Risk:}




\section{Understating Polygenic Information:}

All participants had a good understanding of their breast cancer risk and all recalled their PRS category. However, most participants recalled the risk category assigned to their polygenic risk (high and low) rather than the numerical figures provided.

"Well from the gene, from what they tested I was a low risk...I can't remember what they were called now but I was low in having those." (Donna, 40 years, Affected/Low PRS) "what I took away from that was I guess I'm higher [breast cancer risk] than most people..." (Emma, 50 years, Unaffected/High PRS)

Most participants, including those who had not attended an FCC prior to the study, demonstrated broad knowledge of concepts related to polygenic information and were able to accurately describe the implications of their PRS. For example, participants with a high PRS accurately reflected that they were not at 'high risk' when compared to women with $B R C A 1 / 2$ pathogenic variants.

“...depending on whether or not you had any, some, moderate or a large number of these [SNPs]...that would give you a risk factor as opposed to the general population. And because I have a higher number, I am at a higher risk than the general population, but less than if I had the BRCA gene, of developing breast cancer..." (Sam, 59 years, Affected/High PRS)

Similarly, women with a low PRS recalled that their risk was lower than that of the general population. All women with a low PRS acknowledged their result did not mean "no risk". 
"Well, as I understand it, you can just be unlucky, you know, you could have no genetic risk at all and just be unlucky and get breast cancer, you could have all the genes of breast cancer and be lucky and not get it, you know, it's a roll of the dice really. I'm less likely but that also doesn't mean it's a guarantee. It's not a zero risk." (Jane, 56 years, Unaffected/Low PRS)

Regarding risk to family members, most participants understood the personalized nature of their PRS and correctly described their risk as a combination of maternally and paternally inherited variants. Many participants were relieved by the personalized nature of information pertaining to their breast cancer risk, with many hoping their relatives would have a lower risk for the disease.

“...I suppose the fact that they were saying that, you know the variables can cluster in one person I guess gave me some reassurance that just because I have high risk of something, they [sisters] are not going to as well, and same with my children...." (Melissa, 42 years, Affected/High PRS)

"I do feel it's better for my daughters now as well, given that they've got half of my genes.... and at this stage there isn't any breast cancer on my husband's side, so I sort of feel, breast cancer wise, reasonably confident." (Ashely, 42 years, Unaffected/Low PRS)

Finally, participants demonstrated awareness that this PRS was not associated with changes in ovarian cancer risk. Participants with a high PRS and without a family history of ovarian cancer reported feeling relieved by this information, often comparing it to the ovarian cancer risk associated with $B R C A 1 / 2$ pathogenic variants. 
"Yes so the information that there's no link with ovarian cancer as compared to the BRCA genes. So there was no need for me to worry about ovarian cancer. That's good. ” (Isabel, 49 years, Affected/High PRS)

\section{The multifactorial nature of breast cancer risk:}

Despite all participants recalling their PRS, none felt this was the only contributing factor to their breast cancer. Instead, all participants felt their overall risk was a combination of multiple factors that included genetics, family history, bad luck, and lifestyle factors.

“...genetics are genetics, you know, just because I have a drink of alcohol it's not going to change my - my absolutely genetic makeup, but if I drink alcohol or if I am overweight...I'm creating an environment that cancer is more likely to be attracted to, I guess... if I am fit and don't put alcohol into my system, then I'm - I am maintaining an environment that's less hospitable to cancer" (Amanda, 47 years, Affected/High PRS)

Many women used the multifactorial nature of breast cancer to help them make sense of their PRS and lived experiences of the disease. For example, some unaffected women with a low PRS relied on the multifactorial model of breast cancer to reconcile the differences between their family history and PRS.

"Mum was a senior executive, had very, very stress job. Previously was a smoker, was quite a big drinker. I start to think there's the genetics side of it I suppose in terms of perhaps getting Dad's genes rather than Mum's but also just the outside influences, particularly stress. ” (Carly, 45 years, Unaffected/Low PRS)

\section{Impact on Breast Cancer Risk Management:}


Despite differences in interpretation of breast cancer risk, receiving personalized PRS information appeared to have little impact on participants' risk management behaviors. Instead how participants viewed their risk management strategies was more strongly influenced by their lived experiences and how they made sense of their breast cancer risk. For example, all participants with a low PRS said they would continue following the breast cancer risk management recommended by their clinician. These participants often reflected on their prior experiences with the disease and felt it was important for them to continue with breast screening.

"I think that we just have to keep on the same care plan and just be vigilant as well. So if you see any changes to go and see a doctor." (Mary, 38 years, Unaffected/Low PRS)

Similarly, few participants who received a high PRS said they would change their current breast cancer risk management. These participants felt their PRS had given them greater awareness of their breast cancer risk and highlighted the need to continue with appropriate risk management strategies.

"It's basically made me more aware of now that I really do have to be more proactive in looking after myself, not that I haven't been, but sometimes you slip back into old habits..." (Emily, 57 years, Unaffected/High PRS)

Additionally, these participants felt reassured by their PRS, as it validated their prior risk management decisions.

"Well I guess it just confirmed everything that I'd done, that I'd made the right decisions and that I was, you know, given the right advice." (Sam, 50 years, Affected/High PRS) 


\section{PRS and population screening:}

Finally, participants felt PRS testing should be extended to the general public in order to personalize population screening programs. This sentiment was often based on their lived experiences with breast cancer. For example, affected women who were diagnosed with aggressive cancers before being eligible for population screening felt strongly that polygenic testing would have allowed them access to better screening and earlier diagnosis.

“...I wasn't forty when I discovered my lump, so I didn't even get into the screening...the other implication I guess from some of these kinds of studies is that it helps to target who needs to be part of screening...so if you know there are people that you need to target then that's actually a good thing..." (Melissa, 42 years, Affected/High PRS)

However, when asked about the possibility of reduced screening for women with a low PRS, some participants felt this would be inappropriate and expressed concerns that some women may misinterpret a low PRS to mean "no risk".

"It could also be falsely reassuring, if you say, you know, you get a low result, do people interpret the results of a test like that being more concrete..." (Brittany, 36 years, Unaffected/Low PRS)

Overall, participants felt baseline screening should be available to all, with increased screening offered to those at higher risk. Once again, this response was based on participants' lived experiences with breast cancer, with women who received a low PRS reflecting on their own experiences of uninformative genetic testing result to date. 
“...because people like me who it's not going to crop up on anything you know. I think

the screening should be there for anybody who wants it" (Kelly, 66 years, Affected/Low PRS)

\section{Discussion:}

The results from this study contribute to the emerging body of literature about the experiences of women receiving polygenic breast cancer risk information. This study adds to the literature by comparing the experiences of women at different levels of polygenic risk, and women with and without a personal history of breast cancer.

In line with previous research $[9,10]$, participants who received a high PRS felt positive about receiving their result as it clarified their future risk of developing breast cancer and provided an etiological explanation for affected women. Perhaps not unsurprisingly, those who received a low PRS also felt positive about this result, describing it as "good news". Previous reports have raised concerns that individuals who receive a low PRS may misrepresent this result to mean "no risk" and as influencing negative health behavior [19, 20]. No evidence of this misunderstanding was identified here, with all women demonstrating accurate knowledge of their risk level and implications for risk management. Thus, our findings contradict previous studies by demonstrating that receiving PRS did not negatively impact risk perceptions or health behavior.

In a study of affected women who received high PRS, Young, et al. [9] reported that participants felt reassured by the personalized nature of polygenic risk. Similar findings were identified here, with participants across all risk levels expressing hope that their relatives would have a lower PRS. Interestingly, the personalized nature of PRS also resulted in 
feelings of uncertainty among unaffected women with a low PRS as they struggled to put their result in the context of their family history. This finding highlights a potential challenge for the implementation of polygenic information in clinical genetics services. Historically, genetic services have focused on testing of monogenic risk genes and its familial implications. However, the implementation of polygenic testing will require a shift towards a personalized model of practice that will require a personal evaluation of PRS for each individual [21]. If polygenic testing is to be implemented in clinical practice, a new model of genetic counselling will need to be developed that accounts for monogenic and polygenic risk, as wells additional risk factors such as family history and lifestyle factors. These challenges will be magnified as genetic testing moves into mainstream medicine.

It is well reported that lived experiences have a significant impact on how individuals respond to genetic information [22-24]. Two types of experiential knowledge have been reported to influence understandings of cancer risk and responses to genetic information: embodied and empathetic knowledge [25]. Embodied knowledge is comprised of an individual's personal experiences living with a cancer diagnosis and treatment, while empathetic knowledge is gained by having close contact with others, such as a relative who has been diagnosed with cancer [25]. The impact of experiential knowledge was evident throughout this study with affected and unaffected women reporting different responses to their PRS. For example, only affected women who received a high PRS reported feelings of distress immediately after receiving their result. These participants reflected on the trauma of their breast cancer diagnosis and thus were momentarily distressed by the ongoing risk of the disease. In contrast, no unaffected women who received a high PRS reported feeling distress. These 
women framed their response based on their relatives' experiences with the disease, which in some cases included early detection and positive prognosis. Overall, our findings highlight the need for genetic health professionals to explore their patient's lived experiences as this can significantly alter how they respond and internalize genetic information.

Studies on the utility of polygenic testing have suggested that women in the general population reach the same threshold for population screening at different ages [4]. Thus, polygenic testing has the potential to lead to more effective stratified population screening programs by targeting screening to women with a higher PRS and more limited surveillance for those at lower risk. Implementation of such personalized screening would depend on several factors, including acceptability of risk-based surveillance and individual breast cancer risk perceptions. Previous studies assessing attitudes towards polygenic testing reported that individuals were accepting of a population screening program based on genetic risk, including increased screening for those at higher risk [26-28]. However, there is less support for reducing screening frequency for those at lower risk, with some describing this as discriminatory [26-28]. Similar findings were identified here, including support among women with a high PRS and concerns raised primarily by those with low PRS, with these participants reflecting on their breast cancer history and uninformative genetic testing results. Future research should explore communication of polygenic information and individual responses to this personalized risk, including impact on health behavior, risk perceptions, population-based screening.

This study's strengths include that it represented the views of affected and unaffected women at different levels of polygenic risk. However, the study is limited as the participants who 
were predominantly of Caucasian ancestry and highly educated. In addition, participants were actively engaged in breast cancer research studies and most had received genetic counselling prior to the study, thus they are likely to represent women who are highly motivated and receptive to receiving new genetic information. However, because the study explored the views of those women most likely to seek polygenic risk testing, findings are likely to be transferable to other settings. Nevertheless, findings from this study point to some potential areas for future research including a need to investigate the views of women who decline to receive their PRS and potential issues to consider regarding the implementation of polygenic information for population screening programs.

Polygenic breast cancer information is now being implemented into clinical practice. However, there are limited studies addressing women's responses to receiving polygenic risk information. Our study demonstrated that women at high-risk of breast cancer who previously received uninformative genetic testing result seek to access new genetic information to reduce uncertainty about their level of risk. Furthermore, women responded well to receiving breast cancer PRS, and this information did not appear to have a negative impact on breast cancer risk management.

\section{Acknowledgements:}

We thank all the women who gave their time to participate in the study. We also thank all the clinicians at the FCCs for accommodating this study and arranging appointment for participants to receive their PRS.

\section{Funding:}


This study is supported by a grant from the Cancer Council of New South Wales (ID: 1079897). TY is supported by a National Health and Medical Research Council (NHMRC) and National Breast Cancer Foundation postgraduate scholarship (ID: 1133049), and a Translational Cancer Research Institute PhD Top-up Scholarship. BM is supported by an NHMRC Senior Research Fellowship Level B (ID: 1078523).

\section{Compliance with ethical standards}

\section{Conflict of Interest:}

Prof. Bettina Meiser has a remunerated consultant role with the company Astrazeneca with respect to an unrelated project. 


\section{References:}

1. Bahcall, O., Common variation and heritability estimates for breast, ovarian and prostate cancers. Nature iCOGS, 2013.

2. Michailidou, K., et al., Association analysis identifies 65 new breast cancer risk loci. Nature, 2017. 551(7678): p. 92-94.

3. Michailidou, K., et al., Genome-wide association analysis of more than 120,000 individuals identifies 15 new susceptibility loci for breast cancer. Nat Genet, 2015. 47(4): p. 373-80.

4. Mavaddat, N., et al., Prediction of Breast Cancer Risk Based on Profiling With Common Genetic Variants. Journal of the National Cancer Institute, 2015. 107(5).

5. Sawyer, S., et al., A Role for Common Genomic Variants in the Assessment of Familial Breast Cancer. Journal of Clinical Oncology, 2012. 30(35): p. 4330-4336

6. Mavaddat, N., et al., Polygenic Risk Scores for Prediction of Breast Cancer and Breast Cancer Subtypes. Am J Hum Genet, 2019. 104(1): p. 21-34.

7. Black, M., et al., Validation of a Polygenic $R$ isk Score for Breast Cancer in Unaffected Caucasian Women Referred for Genetic Testing. Journal of Clinical Oncology, 2018. 36. 
8. Hughes, E., et al., Development and validation of a residual risk score to predict breast cancer risk in unaffected women negative for mutations on a multi-gene hereditary cancer panel. Journal of Clinical Oncology, 2017. 35(15_suppl): p. 1579-1579.

9. Young, M.A., et al., Making Sense of SNPs: Women's Understanding and Experiences of Receiving a Personalized Profile of Their Breast Cancer Risks. Journal of Genetic Counseling, 2018. 27(3): p. 702-708.

10. Forrest, L.E., et al., High-risk women's risk perception after receiving personalized polygenic breast cancer risk information. Journal of Community Genetics, 2018.

11. Bancroft, E.K., et al., The psychological impact of undergoing genetic-risk profiling in men with a family history of prostate cancer. Psychooncology, 2015. 24(11): p. 1492-9.

12. Bancroft, E., K., et al., "It's all very well reading the letters in the genome, but it's a long way to being able to write": Men's interpretations of undergoing genetic profiling to determine future risk of prostate cancer. Familial cancer, 2014. 13(4): p. 625-35.

13. Variants in Practice (VIP). 2019; Available from: https://www.petermac.org/research/clinical-research-trials/clinical-research/familialcancer-research-centre/vip-variants.

14. Yanes, T., et al., Psychosocial and behavioral impact of breast cancer risk assessed by testing for common risk variants: protocol of a prospective study. BMC Cancer, 2017. 17(1): p. 491. 
15. Kaur, R., et al., Development and pilot testing of a leaflet informing women with breast cancer about genomic testing for polygenic risk. Fam Cancer, 2018.

16. Liamputtong, P., Qualitative Research Methods. 4th edition ed. 2013, South Melbourne: Oxford University Press.

17. Braun, V. and V. Clarke, Using thematic analysis in psychology. Qualitative Research in Psychology, 2006. 3(2): p. 77-101.

18. Nvivo Qualitative data analysis software. 2016, QSR International Pty Ltd.

19. Smit, A.K., et al., Exploring the Potential Emotional and Behavioural Impact of Providing Personalised Genomic Risk Information to the Public: A Focus Group Study. Public Health Genomics, 2015. 18(5): p. 309-17.

20. Leventhal, K.-G., et al., "Is it Really Worth it to Get Tested?": Primary Care Patients' Impressions of Predictive SNP Testing for Colon Cancer. Journal of Genetic Counseling, 2013. 22(1): p. 138-151.

21. Cox, D.G., et al., Transmission of breast cancer polygenic risk based on single nucleotide polymorphisms. Breast, 2018. 41: p. 14-18.

22. Hesse-Biber, S., The Genetic Testing Experience of BRCA-Positive Women: Deciding Between Surveillance and Surgery. Qual Health Res, 2014. 24(6): p. 773-789. 
23. McAllister, M., Personal theories of inheritance, coping strategies, risk perception and engagement in hereditary non-polyposis colon cancer families offered genetic testing. Clin Genet, 2003. 64(3): p. 179-89.

24. Nusbaum, R., et al., Translational genomic research: Protocol development and initial outcomes following SNP testing for colon cancer risk. Translational Behavioral Medicine, 2013. 3(1): p. 17-29.

25. d'Agincourt-Canning, L., The effect of experiential knowledge on construction of risk perception in hereditary breast/ovarian cancer. J Genet Couns, 2005. 14(1): p. 55-69.

26. Meisel, S.F., et al., Adjusting the frequency of mammography screening on the basis of genetic risk: Attitudes among women in the UK. Breast, 2015. 24(3): p. 237-41.

27. Henneman, L., et al., 'A low risk is still a risk': exploring women's attitudes towards genetic testing for breast cancer susceptibility in order to target disease prevention. Public Health Genomics, 2011. 14(4-5): p. 238-47.

28. Smit, A.K., et al., Exploring the Potential Emotional and Behavioural Impact of Providing Personalised Genomic Risk Information to the Public: A Focus Group Study. Public health genomics, 2015. 18(5): p. 309-17. 
Table 1: Demographic features of participants $(n=21)$

\begin{tabular}{|c|c|c|}
\hline Participant Description & Level & $\mathbf{N}(\%)$ \\
\hline \multirow{5}{*}{ Age } & $30-39$ & $2(10)$ \\
\hline & $40-49$ & $7(33)$ \\
\hline & $50-59$ & $8(38)$ \\
\hline & $60-69$ & $2(10)$ \\
\hline & $>70$ & $2(10)$ \\
\hline \multirow{2}{*}{ Have daughters } & Yes & $13(62)$ \\
\hline & No & $8(38)$ \\
\hline \multirow[t]{3}{*}{ Employment } & Employed & $13(62)$ \\
\hline & Unemployed & $4(19)$ \\
\hline & Retired & $4(19)$ \\
\hline \multirow[t]{2}{*}{ Marital Status } & Single & $5(24)$ \\
\hline & Relationship & $16(76)$ \\
\hline \multirow[t]{3}{*}{ Education Level } & $\begin{array}{l}\text { High School and } \\
\text { Below }\end{array}$ & $1(5)$ \\
\hline & $\begin{array}{l}\text { Professional } \\
\text { Certificate }\end{array}$ & $7(33)$ \\
\hline & $\begin{array}{l}\text { Bachelor's degree } \\
\text { and above }\end{array}$ & $13(62)$ \\
\hline \multirow[t]{2}{*}{ Birthplace } & Australia & $18(86)$ \\
\hline & Other & $3(14)$ \\
\hline \multirow{2}{*}{ Language Spoken at Home } & English & $21(100)$ \\
\hline & Other & 0 \\
\hline \multirow{4}{*}{$\begin{array}{l}\text { Breast Cancer History/PRS } \\
\text { Group }\end{array}$} & High PRS/Affected & $6(28)$ \\
\hline & High PRS/Unaffected & $5(24)$ \\
\hline & Low PRS/Affected & $5(24)$ \\
\hline & Low PRS/Unaffected & $5(24)$ \\
\hline \multirow{4}{*}{$\begin{array}{l}\text { Number of FDR and SDR } \\
\text { diagnosed with breast } \\
\text { cancer }\end{array}$} & 0 & $5(24)$ \\
\hline & 1 & $8(38)$ \\
\hline & 2 & $7(33)$ \\
\hline & $3+$ & $1(5)$ \\
\hline \multirow{2}{*}{ Attended FCC in the past } & Yes & $19(9.5)$ \\
\hline & No & $2(9.5)$ \\
\hline
\end{tabular}

